\title{
40 Años de la Declaración de Alma Ata. Semblanza histórica del desempeño de Trabajo Social en la Atención Primaria de Salud chilena ${ }^{1}$
}

\author{
Patricia Castañeda Meneses ${ }^{2}$, Ketty Cazorla Becerra ${ }^{3}$, \\ Katherine Cuevas Lang ${ }^{4}$
}

\section{RESUMEN}

El presente artículo conmemora los 40 años de la Declaración de la Conferencia Internacional sobre Atención Primaria realizada en Alma AtaURSS en 1978, acuerdo internacional que transformó la atención sanitaria desde un nivel formal de atención, a una estrategia de desarrollo nacional. $\mathrm{Su}$ propósito original fue proporcionar las orientaciones especializadas que permitieran acercarse a la meta de asegurar a las poblaciones de todas las regiones del mundo Salud para Todos en el año 2000. En este marco y tomando como referente central la Declaración de Alma Ata, se presenta una semblanza histórica que permite revisar los principales antecedentes de la Atención Primaria chilena desde inicios de la república a la fecha, visibilizando los aportes profesionales que ha realizado Trabajo Social en el marco sanitario del país.

Palabras clave: historia de la salud pública chilena, Atención Primaria de Salud, política social de salud, Trabajo Social en salud

\section{Years since the declaration of Alma Ata. Historical semblance of social work in primary chilean health care}

\section{ABSTRACT}

This article commemorates the 40th anniversary of the Declaration of the International Conference on Primary Health Care, held at Alma Ata-USSR in 1978, an international agreement that transformed health care from a formal level of attention to a national development strategy. Its original purpose

Artículo recibido el 3/09/2018. Artículo aprobado el 12/12/1018.

Chilena. Académica e Investigadora. Escuela de Trabajo Social. Universidad de Valparaíso. E-mail: patricia.castaneda@uv.cl

3 Chilena. Académica e Investigadora. Escuela de Trabajo Social. Universidad de Valparaíso. E-mail: ketty.cazorla@uv.cl

4 Chilena. Académica e Investigadora. Departamento de Salud Pública. Escuela de Medicina. Universidad de Valparaíso. E-mail: Katherine.cuevas@uv.cl 
was to provide specialized guidelines aimed at ensuring Health for All in all regions of the world by the year 2000. Within this framework, and using the Alma Ata Declaration as a core reference, this article presents a historical semblance to review the background of the Chilean primary healthcare system from the birth of the Republic to present day, and shedding light on the professional contributions made by Social Work within the framework of the country's healthcare system.

Keywords: History of Chilean Public Health, Primary health care, Social Health Policy, Social Work in Health

\section{0 anos da declaração de Alma Ata. Revisão histórica do desempenho do trabalho social nos cuidados primários de saúde chilena}

\section{RESUMO}

O presente artigo comemora os 40 anos da Declaração da Conferência Internacional sobre Cuidados Primários de Saúde realizada em Alma AtaURSS em 1978, acordo internacional que transformou a atenção sanitária desde um nível formal de atenção a uma estratégia de desenvolvimento nacional. Seu propósito original foi fornecer as orientações especializadas que permitissem aproximar-se da meta de assegurar à população de todas as regiões do mundo Saúde para Todos para o ano 2000. Neste cenário e tendo como referente central a Declaração de Alma Ata, apresenta-se uma revisão histórica que permite examinar os principais antecedentes dos Cuidados Primários no Chile desde os inícios da república até hoje, tornando visíveis as contribuições profissionais que tem realizado o Trabalho Social no panorama sanitário do país.

Palavras-chave: História da Saúde Pública chilena, Cuidados Primários de Saúde, Política Social de Saúde, Trabalho Social em Saúde

\section{Presentación}

En el año 1978 se realiza en la ciudad de Alma Ata, URSS, la Conferencia Internacional de Atención Primaria de Salud, organizada por la Organización Mundial de la Salud (OMS) y el Fondo de las Naciones Unidas para la Infancia (UNICEF). Se buscaba como objetivo fortalecer las acciones de salud en el nivel primario de atención, aspirando a transformarlas en una estrategia de desarrollo integral que otorgase garantías en el derecho a la salud en toda población o grupo humano que lo requiriera. Sus postulados fueron sintetizados en el lema Salud para 
Todos en el año 2000 y las orientaciones de la Declaración de Alma Ata, que suscribieron los países asistentes, establecieron la necesidad de un trabajo mancomunado y de largo plazo que permitiese aspirar al cumplimiento de la ambiciosa meta planteada (OPS, 2012).

A 40 años del hito, el presente artículo conmemora el evento en referencia y expone una semblanza histórica de carácter descriptivo que permite visibilizar los principales hitos de salud pública realizados en la temática a nivel nacional. Así, entonces, se presenta un primer apartado que abarca desde los esfuerzos fundacionales iniciales hasta el año 1978, describiendo la base institucional y de gestión sanitaria en torno a la Atención Primaria chilena en dicha etapa histórica. En un segundo apartado se exponen las implicancias derivadas de la Declaración Internacional en el contexto dictatorial que afectaba a Chile en 1978, y en un tercero se revisan las fuertes restricciones que afectaron el desarrollo de la Atención Primaria de Salud durante los años dictatoriales. En un cuarto apartado se describen los esfuerzos realizados durante el periodo de normalización democrática para proyectar la Atención Primaria nacional a un nivel acorde con las exigencias planteadas por Alma Ata. En cada apartado interesa ilustrar en forma específica las principales acciones profesionales llevadas a cabo por Trabajo Social y que contribuyeron al desarrollo de la Atención Primaria en Chile en los diversos contextos temporales. Finalmente, se presenta una reflexión que sintetiza los principales aprendizajes del desempeño profesional en la Atención Primaria de Salud en el periodo 1978-2018.

\section{Antecedentes históricos de la Atención Primaria de Salud en Chile y aportes profesionales de Trabajo Social previos a la Declaración de Alma Ata de 1978}

Los antecedentes históricos de la Atención Primaria chilena reconocen los esfuerzos realizados al inicio de la República por diversas instituciones de beneficencia ligadas preferentemente a ór- 
denes religiosas, que ofrecían servicios sanitarios generales a la población especialmente con carácter curativo asistencial (Tapia, 2015, p.14). Posteriormente, en 1830 se crea la Junta Central de Vacunas, que entrega el servicio de vacunatorio a la población nacional en forma gratuita, con especial atención a la prevención del contagio por epidemias de viruela (Murillo, 1898), y en 1832 se crea la Junta Nacional de Beneficencia y Salud Pública, organismo público destinado a la atención de las necesidades médico-sociales de la población de menores recursos.

Dado que la preocupación central era optimizar los esfuerzos sanitarios a través de la organización profesional de la asistencia social, la Junta Nacional de Beneficencia impulsó la creación de la primera Escuela de Trabajo Social de Chile y América Latina en el año 1925, bajo los auspicios del médico chileno Alejandro del Río. El objetivo central de la escuela fue "formar visitadoras sociales que hicieran posible la sistematización de la asistencia en el país y colaboraran con el médico y los servicios e instituciones de beneficencia en la atención profesional de las necesidades de la población” (Castañeda y Salamé, 2012, p, 26). A contar de dicho año las acciones sanitarias realizadas en el país considerarán en forma permanente el aporte de Trabajo Social en los equipos de salud responsables de implementar las diversas iniciativas, programas y normativas técnicas distintivas de la atención primaria.

Consecuente con lo anterior, la creación del Patronato Nacional de la Infancia a inicios de siglo XX, dirigido por el médico Luis Calvo Mackenna, consideró la presencia de profesionales de Trabajo Social dentro de sus acciones sanitarias. Así entonces, el Patronato fomentó la creación de las Gotas de Leche, organismos abiertos de protección a la infancia, centrados en la lucha contra la mortalidad infantil, que debían estar en coordinación con una maternidad o policlínico para así tener acceso directo a la población en riesgo. La institución establecía en forma explícita que el ingreso al servicio era a través de evaluación social desarrollada por la trabajadora social en domicilio - visitadora social en ese entonces-, a través de una encuesta social que evaluaba los criterios 
de admisión preferentes, que correspondían a "lactantes menores de un mes de vida con alimentación materna, con estado de salud satisfactorio y probabilidades de asistencia regular" (Asociación Chilena de Asistencia Social, 1933, p. 7). La visitadora social, junto con una enfermera visitadora, constituían el equipo profesional que estaba a cargo de la atención de la población infantil en el hogar, en donde realizaban acciones de educación sanitaria y evaluación de condiciones de riesgos de salud, sociales y ambientales, conforme sus ámbitos de competencia profesional. Las mayores amenazas sanitarias materno-infantiles de la época correspondían a desnutrición, tuberculosis, aborto y enfermedades de transmisión sexual (Pemjean, 2011).

El avance en la normativa de salud pública en el país tendría importantes hitos en los años venideros. En 1918 se dictó la Ley $\mathrm{N}^{\circ}$ 3.385, que propuso el primer código sanitario y creó la Dirección General de Sanidad, cuyas atribuciones más relevantes correspondían a la inspección sanitaria, la desinfección pública y los servicios de vacunación, la vigilancia del ejercicio de la medicina y demás ramas del curar, de lazaretos u otros locales destinados a profilaxis; y el tratamiento de enfermedades infecciosas epidémicas (Art. $7^{\circ}$ ). En 1924 se creó el Ministerio de Higiene, Asistencia y Previsión Social, y se organizó la Caja de Seguro Obrero, para cubrir los riesgos de enfermedad, invalidez, vejez y muerte. Quedaron bajo su dependencia todas las estructuras de atención de salud y de previsión social existentes hasta la fecha y que correspondían a Dirección General de Sanidad, Inspección General del Trabajo, Juntas de Beneficencia, Consejo de Habitación Popular, Cajas de Seguro Obrero y de Previsión de Empleados (Jiménez, 2001). En 1931 se crea el Servicio Nacional de Salubridad, en el marco de la dictación de un nuevo código sanitario, que impulsó la creación de centros preventivos Madre y Niño para su atención sanitaria preferente (DFL 226 del 15.05.1931). En 1937 se dictó el Decreto Ley 308, que estableció que el Servicio de Seguro Obrero se hiciera cargo de un servicio de protección de la madre y el niño, incluyendo acciones de medicina preventiva. El mismo año entra 
en vigencia la Ley 6.236, conocida como la Ley Madre y Niño, que inicia la distribución gratuita de leche a la infancia menor de dos años. En este creciente marco legal de protección y fomento de la atención sanitaria materno-infantil, se consolida progresivamente la figura de la visitadora social, como sujeto "capaz de relacionar la medicina con el hogar, y la ciencia con el humano (...) el rostro humano de la ciencia y el Estado, alguien preparada y debidamente remunerada para "visitar" y recorrer los hogares del pueblo" (Pemjeam, 2011, p. 109).

Un importante respaldo de los aportes de Trabajo Social a la salud materno infantil se concreta en 1952, con la promulgación de la Ley 10.383 que crea Servicio Nacional de Salud (SNS), unificando los servicios de salud que estaban radicados a la fecha en distintos servicios y reparticiones públicas. Su objetivo fue definido como "encargado de la protección de la salud por medio de acciones sanitarias y de asistencia social y atenciones médicas preventivas y curativas" (Art.62). El Servicio se orientó a optimizar la cobertura y la capacidad de intervención sobre problemas sanitarios importantes para la época, centrando sus esfuerzos en el Programa Materno Infantil. Así nacen los programas de control de niño sano, control de desnutrición, prevención de diarrea infantil, vacunaciones ampliadas, de control y atención profesional del embarazo y parto, programa de planificación familiar, programa nacional de alimentación complementaria y otras acciones sanitarias asociadas. Las principales estrategias de acción correspondieron a la educación sanitaria y a la participación comunitaria en salud, que incluyó en forma preferente a sectores urbanos de menores ingresos y sectores rurales, procesos que fueron liderados al interior de los equipos sanitarios por profesionales de Trabajo Social. Las acciones se realizaron desde los establecimientos comunales de atención primaria y hospitales tipo 4 o rurales (Jiménez, 2001).

La validación de las estrategias intersectoriales de educación en salud, realizadas por Trabajo Social a la fecha, se vieron confirmadas en 1956, con la creación de la instancia interministerial Comi- 
sión Mixta Salud y Educación, que buscaba la coordinación de los esfuerzos de instituciones de salud y educativas en un mismo territorio, considerando los equipos profesionales de ambos sectores e integrando también a padres de familia y miembros de la comunidad, con la finalidad de propiciar o mejorar las condiciones del ambiente físico, cultural y social en favor de la salud infantil. El Servicio Nacional de Salud instruye a través del Boletín $\mathrm{N}^{\circ} 2$ correspondiente al periodo marzo-abril de 1956, la constitución de la Comisión Mixta de Salud Pública y Educación, a nivel nacional y en cada Centro de Salud del país, correspondiendo a sus principales funciones "el estudio de los problemas de salud en el contexto escolar, la investigación de la protección de la salud del niño y del maestro, la educación para la salud y la proyección de la labor educativa hacia el hogar y la comunidad" (SNS, 1956, p. 39). La labor de coordinación intersectorial canalizó los esfuerzos profesionales de Trabajo Social como articulador de las acciones conjuntas en los establecimientos educacionales ubicados en los territorios locales, con el fin de favorecer especialmente el acceso de la población escolar a las acciones educativas y sanitarias programadas.

A partir del año 1955 comienzan los ciclos de destinaciones de médicos generales de zona y de residencias de especialidades, que permiten dotar de equipos médicos a las zonas más apartadas del país (Jiménez, 2001). En estas acciones se contó con una importante participación de la comunidad, mediante la creación de consejos comunales de salud y consejos paritarios en consultorios y hospitales, acciones respaldadas a través del Decreto del Ministerio de Salud Nº 250 de 1967, y más tarde con la creación de los consejos locales de salud, a través del Decreto Ley 602 del Ministerio de Salud de 1971, permitiendo que la población contribuyera activamente al desarrollo y consolidación de las iniciativas. En este marco, las acciones profesionales de Trabajo Social, orientadas a fortalecer la participación comunitaria en salud, contribuyeron a enriquecer su desempeño, trascendiendo desde una impronta inicial predominantemente asistencialista, hacia una valorización explícita de la promoción y organización social, redefinición que 
ha quedado sintetizada en el concepto de agentes de cambio social que simboliza el trabajo profesional de dicho periodo (Castañeda y Salamé, 2016).

Durante la década de 1960 y hasta septiembre de 1973, las orientaciones ministeriales buscaban consolidar la atención pública en el nivel de salud primaria, priorizando acciones que consideraran la participación comunitaria como parte de la búsqueda de soluciones en recursos, servicios e infraestructura, junto con reconocer a la comunidad como parte activa en los procesos de toma de decisiones sanitarias. Como consecuencia de lo anterior, Trabajo Social asumió un rol central en la vinculación con la organización social, aportando sus avances conceptuales y metodológicos en torno a la promoción social impulsados por los ámbitos profesionales, académicos e institucionales de la época, respaldando una empleabilidad favorable basada en la necesidad de implementar las orientaciones ministeriales en todos los centros de salud primarios del país. Así, se crearon consultorios periféricos en sectores urbanos, que permitieran entregar una respuesta sanitaria oportuna a los masivos procesos migratorios campo/ciudad sucedidos en Chile en las décadas de 1950 y 1960, precipitados por la creciente industrialización de los centros urbanos y que sobrepasaron la infraestructura y los servicios sanitarios disponibles. El sector rural fue apoyado con estaciones médico-rurales y postas rurales, muchas veces gestionadas en cooperación con los recursos de la propia comunidad y sectores patronales que aportaban obras para mejorar la accesibilidad en la atención de salud de la población (Ugarte, 1970). En todos ellos, los equipos de salud consideraban el aporte de Trabajo Social para el desarrollo de acciones directas que priorizaban la participación de la comunidad y la educación en salud.

Con el abrupto cambio de las condiciones institucionales del país, a partir del Golpe de Estado de septiembre de 1973, la salud primaria abandona forzadamente sus actividades vinculadas a la participación social y debe contraerse a sus actividades técnicas asistenciales. En 1979 se inicia un proceso de reforma general al 
sector salud que reorganiza la institucionalidad existente, sentando las bases para el desarrollo mercantil de un sistema mixto público-privado (Tapia, 2015). El nivel primario es descentralizado, traspasando a las municipalidades los activos, recursos financieros, recursos humanos y normas de administración financiera de las estaciones médico-rurales, postas de salud rural y consultorios generales urbanos y rurales del país, manteniendo el Ministerio de Salud la supervisión técnica de sus acciones (DFL N¹-3 de 1980). En este contexto, Trabajo Social debió redefinir forzadamente sus funciones, siendo remitido a una tarea asistencial, centrada principalmente en la calificación previsional y en la administración de las Secciones de Orientación Médico Estadística SOME de los establecimientos de salud primarios (Castañeda y Salamé, 2016).

\section{Declaración de Alma Ata y contexto chileno en 1978}

En 1978 se celebra en Alma-Ata, URSS, la Conferencia Internacional sobre Atención Primaria de Salud, organizada por la Organización Mundial de la Salud (OMS) y el Fondo de las Naciones Unidas para la Infancia (UNICEF). En su Declaración final, la Conferencia plantea como meta la necesidad de alcanzar la Salud para Todos en el año 2000, aportando una renovada definición de la Atención Primaria de Salud, que la transforma desde un nivel formal de atención a una estrategia de desarrollo factible de ser implementada en todas las regiones del mundo (OPS, 2012). Los nuevos términos en que se expresa la definición corresponden a los siguientes:

La Atención Primaria de Salud es la asistencia sanitaria esencial basada en métodos y tecnologías prácticos, científicamente fundados y socialmente aceptables, puesta al alcance de todos los individuos y familias de la comunidad mediante su plena participación y a un costo que la comunidad y el país puedan soportar, en todas y cada una de las etapas de su desarrollo con un espíritu de autorresponsabilidad y autodeterminación. La atención primaria forma parte integrante tanto del sistema 
nacional de salud, del que constituye la función central y el núcleo principal, como del desarrollo social y económico global de la comunidad. Representa el primer nivel de contacto de los individuos, la familia y la comunidad con el sistema nacional de salud, llevando lo más cerca posible la atención de salud al lugar donde residen y trabajan las personas, y constituye el primer elemento de un proceso permanente de asistencia sanitaria (OMS, 2008).

La Declaración tuvo la claridad de definir un conjunto de valores que orientaron el desarrollo sanitario y la organización de los servicios de salud, incorporando la participación social como parte de la estrategia sanitaria. Los objetivos generales correspondían a la equidad en el acceso a la atención y la eficacia en la prestación de los servicios (OMS, 2008).

Estos potentes postulados sanitarios internacionales chocaron con la realidad nacional de ese entonces. Chile se encontraba en plena dictadura y el tema de las violaciones de los derechos humanos, iniciado en 1973, emergía en forma inesperada a través del hallazgo de las osamentas de detenidos desaparecidos en los hornos de cal de la localidad de Lonquén, en la Región Metropolitana, constituyendo un momento emblemático que hizo públicas las prácticas de detención, desaparición e inhumación ilegales realizadas en dictadura, y negadas sistemáticamente por los organismos oficiales (Ahumada y otros, 1989). También ese año se dictaba el Decreto Ley No 2.191, 18 de abril de 1978, que establecía amnistía para las personas autoras, cómplices y encubridoras de hechos delictuosos ocurridos en Chile durante la vigencia del Estado de Sitio entre el 11 de septiembre de 1973 y el 10 de marzo de 1978. En forma paralela, Chile se encontraba en un punto crítico de conflicto con Argentina, por el litigio marítimo sobre la soberanía de las islas Picton, Nueva y Lennox, y espacios marítimos adyacentes ubicados en Canal Beagle (Lacoste, 2004).

En este tenso marco nacional, las posibilidades de la atención primaria de ser resignificada a partir de los lineamientos propuestos 
por Alma Ata estaban lejos de concretarse. Mientras la Declaración Internacional reconocía en su Artículo I la condición de la salud como parte de los Derechos Humanos, en Chile éstos eran sistemáticamente vulnerados, en condiciones de impunidad. A pesar de que el Artículo III planteaba que el pueblo tiene el derecho y el deber de participar individual y colectivamente en la planificación y aplicación de su atención de salud, en Chile la participación social se encontraba absolutamente reprimida y bajo total censura. Cuando el Artículo III de la Declaración proclamaba el valor de la Atención Primaria de Salud como contribución a la Paz Mundial, el país se encontraba al borde de iniciar un conflicto armado con un país vecino, que podría haber generado trágicas consecuencias a la población de ambas naciones. Y mientras el Artículo VIII de Alma Ata convocaba a todos los gobiernos a la formulación de políticas, estrategias y planes de acción nacionales, con objeto de iniciar y mantener la Atención Primaria de Salud como parte de un sistema nacional de salud completo y en coordinación con otros sectores, Chile estaba a punto de comenzar al año siguiente un proceso de descentralización con orientación privatizadora del sistema de atención de salud, a través de la municipalización del nivel primario y de la progresiva incorporación del sector privado en la atención sanitaria de la población de mayores ingresos. Es por lo anterior que, en opinión de profesionales sanitarios expertos a nivel nacional, es posible afirmar que entre 1978 y 1990 los postulados de Alma Ata no tuvieron efectos en la Atención Primaria chilena (López, 2018).

No obstante lo anterior, debe dejarse constancia que los postulados de Alma Ata se implementaban incipientemente en forma paralela fuera de las acciones y políticas oficiales, a través de las organizaciones no gubernamentales y organizaciones solidarias que funcionaban en el país en aquella época, vinculadas con la atención de salud de amplios grupos sociales reprimidos en sus derechos fundamentales y desplazados de sus beneficios previsionales. Estos organismos fueron apoyados por aportes entregados principalmente por la Iglesia católica y otros credos, junto con agencias 
de cooperación internacional, permitiendo mantener las acciones vinculadas a la educación y promoción en salud en poblaciones y territorios focalizados, impidiendo con ello que la tradición de salud pública nacional se discontinuara en forma total. Las acciones se realizaban principalmente a través de policlínicos solidarios, atención materno-infantil, formación de monitores de salud, acompañamiento sanitario rural, capacitación en tecnologías alternativas de apoyo a la subsistencia de la población y rescate de prácticas culturales de salud en sectores campesinos y poblacionales, entre las iniciativas de mayor relevancia realizadas en la época. Especial mención reviste la atención realizada en familiares de víctimas de la represión, mediante estrategias de intervención y acompañamiento individual, familiar y grupal, sentando las bases de la atención en salud mental que se realizaría en las décadas posteriores. En este marco, Trabajo Social, como profesión, aporta técnicamente mediante la formulación, implementación y evaluación de proyectos de acción social y educativa en contextos de resistencia, junto con su permanente participación en los equipos de salud alternativos, en los que contribuyó con su experiencia educativa, organizacional y de apoyo a la participación social en poblaciones urbanas y rurales afectadas directamente por las acciones represivas, y por las continuas crisis económicas que caracterizaron a la década de 1980 .

\section{Desarrollo profesional de Trabajo Social en salud en el marco de Alma Ata en los años dictatoriales. Chile 1978-1990}

Los años dictatoriales afectaron profundamente el desarrollo de la atención primaria chilena, especialmente en su financiamiento y administración. A contar de 1979, la dictadura chilena inició el proceso de municipalización de la salud primaria, situación que fragilizó su financiamiento, dado que se comenzó a realizar por medio de una modalidad de pagos basada en la facturación por atención prestada en establecimientos municipalizados, conocida por su sigla FAPEM. El arancel estaba determinado por 
el tipo de acciones realizadas, valorizando las acciones curativoasistenciales por sobre la promoción, prevención y educación en salud. El monto total de cada establecimiento estaba predefinido, sin hacerse cargo de las acciones excedentes, desfinanciando progresivamente el sistema y provocando serios déficit en inversiones de personal, infraestructura, tecnología, insumos, transportes y comunicaciones. En este marco, originalmente se esperaba contar con aportes complementarios de la población usuaria en lógica de copago y con recursos provenientes de los municipios. La administración municipal enfrentaba su propio déficit, dadas las sucesivas crisis económicas de la década de 1980, generando un escenario adverso que ligó la realidad socioeconómica de cada comuna a la cantidad y calidad de servicios que podía ofrecer en atención primaria, situación especialmente crítica en los municipios que concentraban poblaciones de bajos ingresos, con alta demanda por salud municipal (Sacarpaci, 1988).

Como efecto de estas medidas, las acciones de Trabajo Social vinculadas a participación social y promoción social son desestimadas, dado que no aportaban recursos directos desde las prestaciones establecidas en el FAPEM y no resultaban pertinentes en el marco represivo de la época. Su tarea profesional se desvaloriza fuertemente y su desempeño se focaliza en las tareas de calificación previsional y atención asistencial individual y familiar, a través de la consulta social y la visita domiciliaria. La única actividad de perfil comunitario que se mantiene durante el periodo es la Comisión Mixta Salud-Educación, la que funciona con un marcado perfil sanitario asistencial y con acciones educativas destinadas a grupos estudiantiles focalizados, centradas en prevención de embarazo adolescente y adicciones. Sin embargo, en este estricto marco de restricciones emerge la jefatura del Servicio de Orientación Médica Estadística, SOME, como creciente espacio profesional vinculado a la gestión institucional, dada la directa relación de la función de calificación previsional con los criterios de acceso a la atención de salud pública en sus niveles secundario y terciario. Estos niveles revisten mayor especialización en los servicios 
y prestaciones sanitarias que ofrecen, e inician su proceso de tramitación a partir de la interconsulta de atención primaria, permitiendo el acceso a atención clínica, procedimientos clínicos, hospitalizaciones o exámenes avanzados. Este espacio de gestión fue utilizado frecuentemente por el colectivo profesional para ajustar las estrictas reglas establecidas por las autoridades de la época a las necesidades de la población, realizando acciones en el interlineado de la norma que permitían concretar las situaciones de excepción, posibilitando el acceso a la atención de salud a través de importantes rebajas en el copago y de respaldo a la calificación de gratuidad.

Asimismo, en este periodo y en forma sincrónica a las acciones que realizaban las organizaciones no gubernamentales, Trabajo Social comenzó a realizar de manera exploratoria las primeras atenciones de salud mental de la población en el nivel primario, como respuesta a los crecientes diagnósticos iniciales de depresión y estrés visibilizados en la población, y que carecían de respuestas institucionales en su contención y orientación. Si bien estas intervenciones mantuvieron el carácter asistencial e individual que caracterizaron a la acción profesional en estos años, formalizaron los aportes de la única disciplina de las ciencias sociales que a esa fecha formaba parte de los equipos de atención primaria y que en ese carácter podía hacerse cargo de estas problemáticas, las que fueron aumentando su complejidad en diagnósticos y prevalencias en las décadas posteriores.

Debe reconocerse que, a pesar del adverso contexto de desempeño descrito, la profesión siguió siendo considerada durante estos difíciles años como parte integrante de los equipos de salud, definiendo sus acciones sanitarias distintivas en sus programaciones anuales y siendo respaldados sus aportes en las emergentes áreas de gestión y salud mental desde los niveles técnicos ministeriales. Si bien se reconoce que en algunos servicios sus plazas disminuyeron, Trabajo Social nunca fue eliminado totalmente de la dotación de los equipos de atención primaria de salud a lo largo del país. 


\section{Desarrollo profesional de Trabajo Social en Salud en el marco de Alma Ata a partir del retorno a la Democracia. Chile 1990-2018}

A partir de marzo de 1990, se define como una de las prioridades nacionales el fortalecimiento de la salud pública, considerando dentro de las medidas iniciales el restablecimiento de la gratuidad de la atención primaria a su población beneficiaria (MINSAL, 1992). Asimismo, se destinan recursos para la conformación de equipos de reforzamiento psicosocial para la atención primaria, constituidos por profesionales de Trabajo Social, Psicología y Obstetricia (MINSAL, 1993). Las acciones de los equipos interdisciplinarios psicosociales consideraron la intervención en salud mental en sus modalidades individual y grupal, focalizada en la población femenina y sus problemáticas asociadas de violencia intrafamiliar y depresión, como respuesta a las demandas de mayor recurrencia en la época. También sus tareas se enfocaron en forma prioritaria a la promoción de la participación ciudadana, con especial atención en la recomposición de los vínculos entre las organizaciones sociales y los equipos de salud, relación resentida por los largos años dictatoriales que impidieron la comunicación entre ambas instancias y cuyos efectos se amplificaban debido a las fuertes restricciones que había enfrentado la atención primaria a partir del proceso de municipalización.

En el contexto comunitario, la participación en salud es revalorizada desde instancias técnicas y políticas, expresándose en la decidida reactivación de los Consejos Locales de Salud, que restablecen la histórica relación de colaboración entre los equipos de salud y la comunidad en las problemáticas de salud de su territorio. En forma convergente, las Comisiones Mixtas Salud-Educación enriquecen su quehacer, sumando a su tarea asistencial renovadas acciones de promoción de salud, que aportan nuevos referentes a los contenidos y a las acciones intersectoriales que se desarrollan en forma amplia a través de convocatorias abiertas que involucran a la totalidad de las comunidades de los establecimientos educacionales adscritos al centro de salud primario. En ambas instancias comu- 
nitarias, Trabajo Social asume su liderazgo técnico en el desarrollo e implementación de las iniciativas conjuntas llevadas a cabo entre los equipos de salud y la población organizada. A fin de optimizar su desempeño en estas responsabilidades, se redefine la tarea de calificación previsional que había concentrado los mayores esfuerzos de horas profesionales durante el periodo dictatorial, permitiendo la delegación de los procedimientos formales de calificación definidos por ley a cargo de equipos técnicos de apoyo para la calificación de derechos que recibieron capacitación especializada para tal fin, reservando para la atención de Trabajo Social las situaciones de mayor complejidad y de excepción, junto con las tareas de supervisión y evaluación del sistema (MINSAL, 1992).

La revitalización de las acciones de educación en salud a partir del año 1990 abarca todo el espectro de temáticas sanitarias y de poblaciones involucradas, ocupando Trabajo Social una posición de liderazgo en atención primaria, en el marco de diagnóstico de necesidades educativas, organización de convocatorias en grupos de interés e implementación de acciones formativas en salud. Esta tarea recibe un importante respaldo institucional a contar del año 1998, cuando el Ministerio de Salud crea una unidad técnica encargada de la Promoción de Salud, como respuesta a las orientaciones recibidas desde la Organización Mundial de la Salud en torno a la promoción de estilos de vida saludables a nivel comunitario, que permitan intervenir en los determinantes sociales de la salud y movilizar recursos sociales para enfrentar los riesgos asociados, especialmente en el desarrollo de enfermedades crónicas no transmisibles (Salinas y Vio, 2002). La promoción de salud ha generado en forma progresiva importantes iniciativas sanitarias traducidas en planes locales, acciones educativas intersectoriales, valoración de buenas prácticas, acciones de difusión y encuentros temáticos a nivel nacional, regional y comunal, permitiendo resignificar los históricos aportes de Trabajo Social desde el ámbito de la promoción social hacia la promoción de la salud, en el marco de las actuales oportunidades educativas que representa la realidad sociosanitaria chilena. 
En el ámbito de la salud mental, los incipientes esfuerzos realizados por Trabajo Social durante la década de 1980, en ámbitos gubernamentales y no gubernamentales, aportan a la formulación de las acciones del Primer Plan Nacional de Salud Mental de 1993, que define en sus estrategias el fortalecimiento de la Atención Primaria de Salud como una instancia territorial y comunitaria para el abordaje de problemas de salud mental, los que a la fecha eran concebidos desde lógicas asistencialistas y hospitalo-centristas. Los equipos de salud locales son capacitados en la atención de problemas de salud mental definidos en torno a los trastornos emocionales y violencia intrafamiliar, $y$, dentro de este marco, Trabajo Social traduce su nueva formación en el despliegue de intervenciones ligadas al tratamiento de las problemáticas de salud mental bajo enfoques individuales y familiares. Asimismo, desarrolla intervenciones preventivas en modalidad grupal en espacios escolares, aproximándose con ello a abordajes intersectoriales que luego han sido subrayados en políticas públicas posteriores (Minoletti, Rojas y Horvitz-Lennon, 2012).

La necesidad de robustecer los equipos de salud mental en atención primaria se traduce en la incorporación de profesionales del área de la psicología para la constitución de duplas psicosociales, en alianza con Trabajo Social. Estas duplas ingresan mayoritariamente a contar de 1999, a partir de nuevas indicaciones del Ministerio de Salud que regulan la organización de una renovada red de prestaciones en la salud mental, especialmente adscritas a la atención primaria municipal de la época. Esta norma contempla la constitución inicial de duplas psicosociales a las que se sumarán más equipos multidisciplinares adscritos al Programa de Salud Mental de Atención Primaria, enmarcado en el Segundo Plan Nacional de Salud Mental y Psiquiatría del año 2000. Este plan tiene por principal objetivo propiciar un enfoque biopsicosocial, basado en la organización de redes de salud mental y servicios comunitarios, definiendo para Trabajo Social oportunidades de intervención en cada una de las temáticas vinculadas a promo- 
ción y prevención en salud mental contempladas y que consideran trastornos de salud mental asociados con la violencia (maltrato infantil, violencia intrafamiliar y represión política en el periodo 1973-1990), trastornos de hiperactividad y de la atención en niños y adolescentes, depresión, esquizofrenia, enfermedad de Alzheimer y otras formas de demencia, y el abuso y la dependencia del alcohol y las drogas (Minoletti y Zaccaria, 2005).

A fines de 1990 se constituyen los primeros Centros de Salud Mental Comunitarios (CSMC), definidos como unidades descentralizadas de atención especializada, ubicados en el territorio de mayor riesgo social e integrados por organizaciones locales y redes comunitarias. Su dotación interdisciplinaria contempla profesionales de Trabajo Social, que se focalizan en la intervención en las adicciones como una de las primeras oportunidades para instalar duplas psicosociales en la territorialidad específica, siendo financiados por el Comisión Nacional de Control de Consumo de Estupefacientes, CONACE (actual Servicio Nacional para la Prevención y Rehabilitación del Consumo de Drogas y Alcohol, SENDA) y el Servicio Nacional de Menores, SENAME. Sobre esta base, se transita a la conformación de los primeros Centros Comunitarios de Salud Mental COSAM, en los que la labor profesional se orienta a la intervención en trastornos mentales severos y persistentes a nivel individual, familiar y del entorno inmediato, favoreciendo la inclusión social y proveyendo las condiciones que contribuyan al pleno ejercicio de los derechos de las personas y su respectivo bienestar social (MINSAL, 2017).

Indudablemente, uno de los aspectos más destacados del periodo corresponde al acceso de Trabajo Social al desempeño en los cargos de dirección de establecimientos de Atención Primaria, respaldado por la promulgación del Estatuto de Atención Primaria en 1995. El cuerpo legal normó la administración, financiamiento y coordinación del nivel primario desde los principios de descentralización y desconcentración, junto con regular la relación laboral, la carrera funcionaria y los deberes y derechos del personal, fuertemente afectados con el proceso de municipalización. Las 
principales demandas del cargo están asociadas a la planificación anual del establecimiento para el cumplimiento oportuno de sus compromisos, metas y objetivos de trabajo definidos para su población y territorio. También comprenden los ámbitos de gestión clínica, gestión en red, gestión del modelo de atención, gestión administrativa, gestión del recurso humano, gestión financiera, gestión participativa y de la población usuaria. Todas estas demandas deben ser resueltas desde un manejo avanzado de un conjunto de competencias técnicas, de gestión y personales, que coinciden en forma central con el perfil profesional de Trabajo Social en Atención Primaria de Salud, permitiendo reconocer a la fecha el desarrollo de experiencias exitosas de dirección y gestión de establecimientos de salud primarios por parte del colectivo profesional.

A contar 2005, en el marco de la Reforma de Salud, se impulsa el Modelo de Atención Integral en Salud Familiar y Comunitaria (MINSAL, 2012), que define en el centro del quehacer sanitario y como eje del desarrollo social a la población usuaria. En consecuencia, se transforman progresivamente los establecimientos de atención primaria a Centros de Salud Familiar y Comunitaria, CESFAM, focalizando territorios y poblaciones específicas a través de los Centros Comunitarios de Salud Familiar, CECOSF. Estos renovados modelos sanitarios posicionan a Trabajo Social en el eje central de la relación que establece la atención primaria con las familias, comunidades y territorios definidos, aportando con su acervo profesional especializado al acompañamiento del ciclo vital familiar desde la perspectiva social. También la Reforma de Salud aportó nuevos sistemas y procedimientos de trabajo a través de plataformas tecnológicas y agendas en línea, que aportaron mayor operatividad en la gestión de la Sección de Orientación Médico Estadística, SOME, la que, a pesar de enfrentar una progresiva especialización, permanece bajo la responsabilidad profesional de Trabajo Social en diversos establecimientos de atención primaria.

A contar de 2012 entra en vigor la Ley $N^{\circ} 20.584$, que explícita los derechos y deberes de la población usuaria, considerando especialmente los relacionados con la dignidad, la autonomía y la 
información, como base para la participación en salud. Una de sus principales expresiones corresponde a la implementación de las Oficinas de Informaciones, Sugerencias y Reclamos OIRS en cada establecimiento de atención primaria, instancia que en muchas ocasiones es responsabilidad profesional de Trabajo Social, reconociendo con ello su pertinencia técnica en la atención social y su adecuado manejo de situaciones en contextos de conflicto. Complementariamente, se realizan esfuerzos para acreditar condiciones de calidad en los servicios entregados por los establecimientos de salud, tarea que también en ocasiones ha sido asumida por profesionales de Trabajo Social, evidenciando su experticia en ámbitos de gestión, en los que se conjuga la exigencia de cumplimiento de estándares sanitarios, junto con el manejo de equipos que deben organizar sus tareas en torno al cumplimiento de objetivos institucionales comunes.

En la proyección del trabajo profesional en la atención primaria en los años futuros, destaca el creciente desarrollo de experiencias de apoyo individual y familiar en el marco de pacientes afectados por problemas de movilidad o enfermedades terminales (Castañeda y Cazorla, 2017) y el acompañamiento de procesos educativos ligados a los cuidados requeridos para un envejecimiento activo y saludable (MINSAL, 2014), acciones que reflejan la creciente complejidad de la realidad demográfica y epidemiológica nacional en la búsqueda de nuevos repertorios de intervención sanitaria de Trabajo Social, que posean la capacidad de aportar respuestas a las demandas emergentes de la población. Asimismo, el desarrollo de líneas de trabajo vinculadas a la salud intercultural, en conjunto con comunidades y asociaciones indígenas (MINSAL, 2017), el acompañamiento a poblaciones migrantes y la inclusión del enfoque de género (MINSAL, 2018), y el reconocimiento del aporte de las medicinas complementarias alternativas en la atención de salud (MINSAL, 2013), enriquecen las miradas sanitarias y valorizan el aporte especializado de la principal profesión social con que cuentan los equipos de atención primaria del país, transformando a Trabajo Social en la principal articuladora del trabajo 
sanitario respecto de los aspectos socioculturales emergentes que caracterizan la sociedad nacional contemporánea.

\section{Reflexiones Finales}

La Declaración de Alma Ata de 1978 constituye un importante punto de inflexión en las oportunidades que representa la Atención Primaria de Salud en el desarrollo nacional, en la medida en que sus postulados valorizan los esfuerzos destinados a la organización y educación en salud como mecanismo central de mejora de los indicadores sanitarios nacionales. En ese marco, la histórica pertenencia de Trabajo Social a los equipos de Atención Primaria de Salud se vio fortalecida, al valorizarse sus competencias profesionales a partir del aporte social especializado que representa la profesión para el desarrollo sanitario del país.

A pesar del desfase en la aplicación plena de sus orientaciones a contar del año 1978, debido al escenario dictatorial que atravesó el país, en la medida en que las condiciones democráticas fueron normalizándose, los saberes profesionales de Trabajo Social en torno a la asistencia, la promoción social y la gestión social han sido revalorizados e integrados plenamente a las acciones de la política social que impulsa la atención primaria chilena en sus diversas líneas de acción.

No obstante debe reconocerse, como marco general, que la trayectoria de 40 años de la Declaración de Alma Ata en Chile está atravesada por la profunda tensión, derivada de un modelo de atención primaria diseñado en el contexto de un sistema mixto público-privado, que ha obligado a realizar esfuerzos permanentes para mantener los favorables indicadores sanitarios alcanzados en décadas pasadas, a pesar del acotado financiamiento sectorial y la creciente complejidad social que afecta la capacidad resolutiva del nivel primario, ocasionando importantes brechas en cobertura, calidad y oportunidad de la atención. Así, entonces, la atención primaria enfrenta, desde sus limitados recursos sectoriales y comunales, las crecientes demandas derivadas de la población de 
menores ingresos, envejecida, migrante, en condiciones de cesantía o de retiro laboral que acude regularmente a sus servicios. Para Trabajo Social esta tensión es una condición de desempeño permanente a la que concurre amplificando los aportes que representa el trabajo conjunto a través de la acción intersectorial, junto con valorizar los esfuerzos organizados de las comunidades locales, que plantean urgentes necesidades sanitarias y aportan con soluciones creativas o reivindicativas en vistas a mejorar las condiciones de salud a nivel comunal.

Asimismo, como reflexión profesional del periodo 1978-2018, se destaca el importante contraste de la valoración de los aportes de Trabajo Social a la Atención Primaria de Salud, directamente asociada a las orientaciones de la política sectorial respecto a las estrategias de educación y participación en salud. Así entonces, cuando las orientaciones y normativas técnicas censuraban dichas estrategias conforme el marco dictatorial que las formulaba, el aporte profesional se replegó a la implementación de las acciones sanitarias de carácter asistencial, desvalorizándose su aporte especializado a nivel de salud comunitaria. Por el contrario, cuando dichas orientaciones formuladas en marcos de progresiva normalidad democrática han respaldado nuevamente las estrategias de carácter socioeducativo y participativo, se observa un importante despliegue profesional que sitúa a Trabajo Social en posiciones de liderazgo técnico frente a la estrategia de atención primaria, respaldada en el dominio avanzado que posee la profesión respecto de las dimensiones individuales, familiares, comunitarias, organizacionales, sociales y culturales asociadas al fenómeno de la salud y la enfermedad.

A modo de corolario, puede plantearse que, si bien la meta original de Salud para Todos en el año 2000 no se cumplió en forma plena a nivel nacional ni a nivel internacional, quedando pendientes importantes brechas en la satisfacción de las necesidades sanitarias de la población, la resignificación de su lema en una permanente demanda por Salud para Todos(as), representa la renovación de los esfuerzos de los equipos de salud de atención pri- 
maria en seguir orientando su quehacer en la mejora en cobertura, accesibilidad y calidad de los servicios y prestaciones que su impronta representa. En esos esfuerzos se suma Trabajo Social como la profesión de las ciencias sociales que ha acompañado, desde su fundación en el año 1925, los afanes de la salud pública y de la Atención Primaria de Salud chilena.

\section{Bibliografía}

Ahumada, E., Atria, R., Egaña, J., Góngora, A., Quesney, C., Saball, G. y Villalobos, G. (1989). Chile: La memoria prohibida. Las violaciones a los Derechos Humanos 1973-1983. Santiago de Chile: Editorial Pehuén.

Asociación Chilena de Asistencia Social. (1933). Principios básicos para la organización y el funcionamiento de las Gotas de Leche. Santiago de Chile: Imprenta Universitaria. Recuperado de http://www.memoriachilena.cl/archivos2/pdfs/ MC0053015.pdf

Castañeda, P. y Cazorla, K. (2017) Cuidar a quien cuida. Sistematización de las fases de cuidado familiar en salud y sus oportunidades de intervención para Trabajo Social. Revista Cuaderno de Trabajo Social. Escuela de Trabajo Social. Universidad Tecnológica Metropolitana, 9(1), 68-85. Recuperado de https://cuadernots.utem.cl/?p=165

Castañeda, P. y Salamé, A. M. (2012). Profesionalidad del Trabajo Social chileno. Tradición y Transformación. Temuco: Ediciones Universidad de La Frontera.

Castañeda, P. y Salamé, A. M. (2016) Trabajo social chileno y dictadura militar. Memoria profesional predictatorial. Participación social en salud, período 1960-1973. Revista Perspectivas. Escuela de Trabajo Social. Universidad Católica Cardenal Silva Henríquez, (27), 29-57. Recuperado de http:// ediciones.ucsh.cl/ojs/index.php/Perspectivas/article/ view/410/360

Jiménez, J. (2001). Artículo Política y organizaciones de salud en chile. Reflexiones históricas. Ars Médica. Revista de ciencias médicas. Universidad Católica de Chile, 30(2). Recuperado de http://www.arsmedica.cl/index.php/MED/article/ view/309/240

Lacoste, P. (2004). La disputa por el Beagle y el papel de los actores no estatales argentinos. Universum (Talca), 19(1), 86-109. Recuperado de https://dx.doi.org/10.4067/ S07183762004000100005 
López, M. (2018). ¿Salud para todos? La atención primaria de salud en Chile y 40 años de Alma Ata. 1978-2018. Santiago de Chile: Ministerio de Salud. Recuperado de http:// www.repositoriodigital.minsal.cl/bitstream/handle/2015/418/39476\%20Libro\%20salud\%20para\%20todos\%20CC0903.pdf? sequence $=3$ \&isAllowed $=y$

MINSAL. (1992). Norma General Administrativa $N^{\circ} 3$. Calificación de gratuidad. Resolución Exenta $N^{\circ} 410$. Santiago de Chile: Ministerio de Salud, Departamento de Asesoría Jurídica.

MINSAL. (1993). Políticas y Plan de Salud Mental. Santiago de Chile: Ministerio de Salud.

MINSAL. (2012). Orientaciones para la implementación del modelo de atención integral de salud familiar y comunitaria. Santiago de Chile: Subsecretaría de Redes Asistenciales, División de Atención Primaria. Recuperado de http://www.minsal.cl/ portal/url/item/e7b24eef3e5cb5d1e0400101650128e9.pdf

MINSAL. (2013). Estudio para validar un protocolo aplicable a las medicinas complementarias y alternativas que se implementan en las redes asistenciales. Instructivo de Aplicación. Santiago de Chile: Ministerio de Salud. Recuperado de http://163.247.80.211/estadistica/Estadistica/2014/FORMATOS\%20REM/MEDICINA\%20COMPLEMENTARIA $\% 20$ \% $20 \mathrm{WCH} /$ INSTRUCTIVO\%20MEDICINA\%20COMPLEMENTARIA\%202014.pdf

MINSAL. (2014). Programa Nacional de Salud de las personas adultas mayores. Santiago de Chile: Ministerio de Salud. Recuperado de http://www.minsal.cl/sites/default/files/files/ Borrador\%20documento\%20Programa\%20Nacional\%20 de\%20Personas\%20Adultas\%20Mayores-\%2004-03_14. pdf

MINSAL. (2017). Modelo de Gestión Centro de Salud Mental Comunitaria. Santiago de Chile: Ministerio de Salud. Recuperado de http://www.minsal.cl/wp-content/uploads/2015/12/ Modelo-de-Gestion-CSMC-9-febrero-172.pdf

MINSAL. (2017). Plan para Pueblos Indígenas. Proyecto de Apoyo al Sector Salud MINSAL - Banco Mundial. Santiago de Chile: Ministerio de Salud. Recuperado de http://www.minsal. cl/wp-content/uploads/2017/04/PPI-_Chile_HESSP-7de-abril-2017-MINSAL-OK.pdf

MINSAL. (2018). Orientaciones para la Planificación y Programación en Red. Santiago de Chile: Ministerio de Salud, Subsecretaría de Redes Asistenciales. Recuperado de http://www.minsal.cl/wp-content/uploads/2015/10/Orientaciones-parala-planificacion-y-programacion-en-red-2018.pdf 
Minoletti, A. y Zaccaria, A. (2005). Plan Nacional de Salud Mental en Chile: 10 años de experiencia. Revista Panamericana de Salud, 18(4-5), 346-358. Recuperado de https://www.scielosp.org/scielo.php?pid=S102049892005000900015\&script=sci_arttext

Minoletti, A., Rojas, G. y Horvitz-Lennon, M. (2012). Salud mental en atención primaria en Chile: aprendizajes para Latinoamérica. Cadernos Saúde Coletiva, 20(4), 440-447. Recuperado de http://www.scielo.br/scielo.php?pid=S1414462X2012000400006\&script=sci_abstract\&tlng=es

Murillo A. (1898). El servicio de vacuna en Chile. Santiago de Chile: Imprenta de Emilio Pérez. Recuperado de http://www.memoriachilena.cl/archivos2/pdfs/mc0027480.pdf

OMS. (2008). Regreso a Alma Ata. Organización Mundial de la Salud. Recuperado de http://www.who.int/dg/20080915/es/

OPS. (2012) Declaración de Alma-Ata. Conferencia Internacional sobre Atención Primaria de Salud. Alma-Ata, URSS, 6-12 de septiembre de 1978. Recuperado de http://www.paho.org/ spanish/dd/pin/alma_ata_declaracion.htm

Pemjean, I. (2011). Una historia con olor a leche: de la desnutrición a la obesidad, políticas públicas e ideologías de género. Revista Punto Género (1), 103-124. Recuperado de https://revistapuntogenero.uchile.cl/index.php/RPG/article/download/16825/17521/

República de Chile. (1918). Ley No 3385 Código Sanitario. Santiago de Chile: Ministerio del Interior. Recuperado de http://bcn. cl/1wzt7

República de Chile. (1931). DFL 226 Código Sanitario. Santiago de Chile: Ministerio de Bienestar Social. Recuperado de http:// bcn.cl/1v23c

República de Chile. (1937). Modifica la Ley 4.054. Santiago de Chile: Ministerio de Salubridad, Previsión y Asistencia Social. Recuperado de http://bcn.cl/1vbn5

República de Chile. (1952). Ley No 10.383. Modifica la Ley 4.054 relacionada con el Seguro Obligatorio. Santiago de Chile: Ministerio de Salubridad, Previsión y Asistencia Social. Recuperado de http://bcn.cl/1uzdg

República de Chile. (1967). Decreto 250. Crea Consejos Comunitarios de Salud. Santiago de Chile: Ministerio de Salud. Recuperado de http://bcn.cl/24e5x

República de Chile. (1971). Decreto 602. Crea Consejos Locales de Salud. Santiago de Chile: Ministerio de Salud. Recuperado de http://bcn.cl/21a2l 
República de Chile. (1978). Decreto Ley No 2.191. Concede amnistía a las personas que indica por los delitos que señala. Santiago de Chile: Ministerio del Interior. Recuperado de http://bcn. $\mathrm{cl} / 1 \mathrm{v} 3 \mathrm{xd}$

República de Chile. (1980). Decreto con Fuerza de Ley $N^{\circ}$ 1-3, que reglamenta Aplicación del Inciso Tercero del Artículo 38 del Decreto Ley $N^{\circ} 3.063$ de 1979. Santiago de Chile: Ministerio del Interior. Recuperado de http://bcn.cl/1vhvk

República de Chile. (1995). Ley $N^{\circ} 19.378$, que establece Estatuto de Atención Primaria de Salud Municipal. Santiago de Chile: Ministerio de Salud. Recuperado de www.bcn.cl/historiadelaley/nc/historia-de-la-ley/7114/

República de Chile. (2012). Ley No 20.584. Regula los derechos y deberes que tienen las personas en relación con acciones vinculadas a su atención en salud. Santiago de Chile: Ministerio de Salud. Recuperado de http://bcn.cl/1uw7l

Sacarpaci J. (1988). Salud y Régimen Militar. Santiago de Chile: Colectivo de Atención Primaria.

Salinas, J. y Vio, F. (2002). Promoción de la salud en Chile. Revista chilena de nutrición, 29 (Supl. 1), 164-173. Recuperado de https://dx.doi.org/10.4067/S0717-75182002029100001

Servicio Nacional de Salud. (1956). Boletín SNS, 2(2). Santiago de Chile: SNS. Recuperado de http://www.bibliotecaminsal.cl/wp/ wp-ontent/uploads/2013/08/Boletin-mar-abr-1956.pdf

Tapia J. (2015). Revisión histórica y legislativa del Derecho a la Salud en Chile. (Memoria para optar al Grado de Licenciado en Ciencias Jurídicas y Sociales). Universidad de Chile, Facultad de Derecho, Departamento de Derecho Público, Santiago de Chile. Recuperado de http://repositorio.uchile.cl/bitstream/handle/2250/130057/Revisi\%C3\%B3nhist\%C3\%B3rica-y-legislativa-del-derecho-a-la-salud-enChile.pdf? sequence $=1$

Ugarte J. (1970) Artículo Salud y Comunidad. Cuadernos Médico Sociales, 3, 31-32. Recuperado de http://cms.colegiomedico.cl/ Magazine/1970/11/3/11_3_8.pdf 